\title{
Review \\ I Believe I Can Fly!: Use of Drosophila as a Model Organism in Neuropsychopharmacology Research
}

\author{
Anjana S Narayanan' and Adrian Rothenfluh*,1,2 \\ 'Department of Psychiatry, UT Southwestern Medical Center, Dallas, TX, USA; ${ }^{2}$ Program in Neuroscience, UT Southwestern Medical Center, \\ Dallas, TX, USA
}

\begin{abstract}
Neuropsychiatric disorders are of complex etiology, often including a large genetic component. In order to help identify and study the molecular and physiological mechanisms that such genes participate in, numerous animal models have been established in a variety of species. Over the past decade, this has increasingly included the vinegar fly, Drosophila melanogaster. Here, we outline why we study an invertebrate organism in the context of neuropsychiatric disorders, and we discuss how we can gain insight from studies in Drosophila. We focus on a few disorders and findings to make the larger point that modeling these diseases in flies can have both mechanistic and predictive validity. Highlighting some translational examples, we underline the fact that their brains works more like ours than one would have anticipated.

Neuropsychopharmacology (2016) 4I, |439-1446; doi:I0.I038/npp.20I5.322; published online I8 November 2015
\end{abstract}

\section{INTRODUCTION}

Progress toward identifying and understanding the mechanistic underpinnings of human neuropsychiatric disorders has been slow. This includes the halting development of novel and efficacious drugs to treat these diseases. Genome-wide association studies initially held promise in identifying the genetic factors contributing to the development of these disorders, but for various reasons, they have not yielded as much insight as initially hoped (Alaerts and Del-Favero, 2009; Iyengar and Elston, 2007). Pitfalls have included locus and allelic heterogeneity, involvement of environmental factors, the probable contribution of a mixture of common and rare variants, and the lack of statistical power in analyzing genome-wide single-nucleotide polymorphisms (SNPs). Another confounding variable is how the expression of each of these genes is regulated by epigenetic mechanisms, such as DNA methylation, or histone modification. Recently, sequencing all the protein-coding regions of DNA from afflicted individuals (also known as exome sequencing) has led to the identification of numerous novel gene variants linked with diseases, including autism spectrum disorders (ASD; Sanders et al, 2012). However, even if we were to succeed and isolate all estimated 1000 genes that contribute to ASD, we still would not know the physiological processes,

\footnotetext{
*Correspondence: Dr A Rothenfluh, Department of Psychiatry, UT Southwestern Medical Center, 5323 Harry Hines Boulevard, Dallas, TX 75390-9|27, USA, Tel: + | 214648 ||69,

E-mail: adrian.rothenfluh@UTSouthwestern.edu

Received 9 January 2014; revised 14 October 2015; accepted 16 October 2015; accepted article preview online 30 October 2015
}

and the underlying molecular framework, that these genes are acting in. Therefore, animal models that can be manipulated at the molecular level will remain essential for the understanding of these disease mechanisms.

What makes for a valid model of human neuropsychiatric disorders? Commonly, people consider three domains of validity. (1) Face validity that asks whether the models 'look like' the disease, that is, whether it recapitulates salient features or characteristic symptoms of the human affliction. (2) Mechanistic validity that asks whether the same mechanisms, including genetic or environmental perturbations, can cause the model to resemble/express the disease state. (3) Predictive validity that asks whether the disease model can be perturbed or treated, including with drugs, to ameliorate its symptoms. Ideally, one would like one's model to be valid in all three domains. This is often hard to achieve, because the model may be inadequate and therefore only achieve subsets of these three domains of validity. In addition to any obstacles being posed by the model itself, there is, however, a fundamental stumbling block toward achieving validity in all domains: the targets in these three domains to achieve and model the human condition may not be well defined, or even known. For face validity, the presentation and diagnosis of the disease may be fluid, and show substantial overlap with other syndromes. Furthermore, no biological biomarkers are available to objectively diagnose the disease. Often, we also know little about the causes of the disease, thus making achievement of mechanistic validity difficult to ascertain. Finally, treatments are sparsely available and are often only partially effective, thus hampering the assessment of predictive validity. 


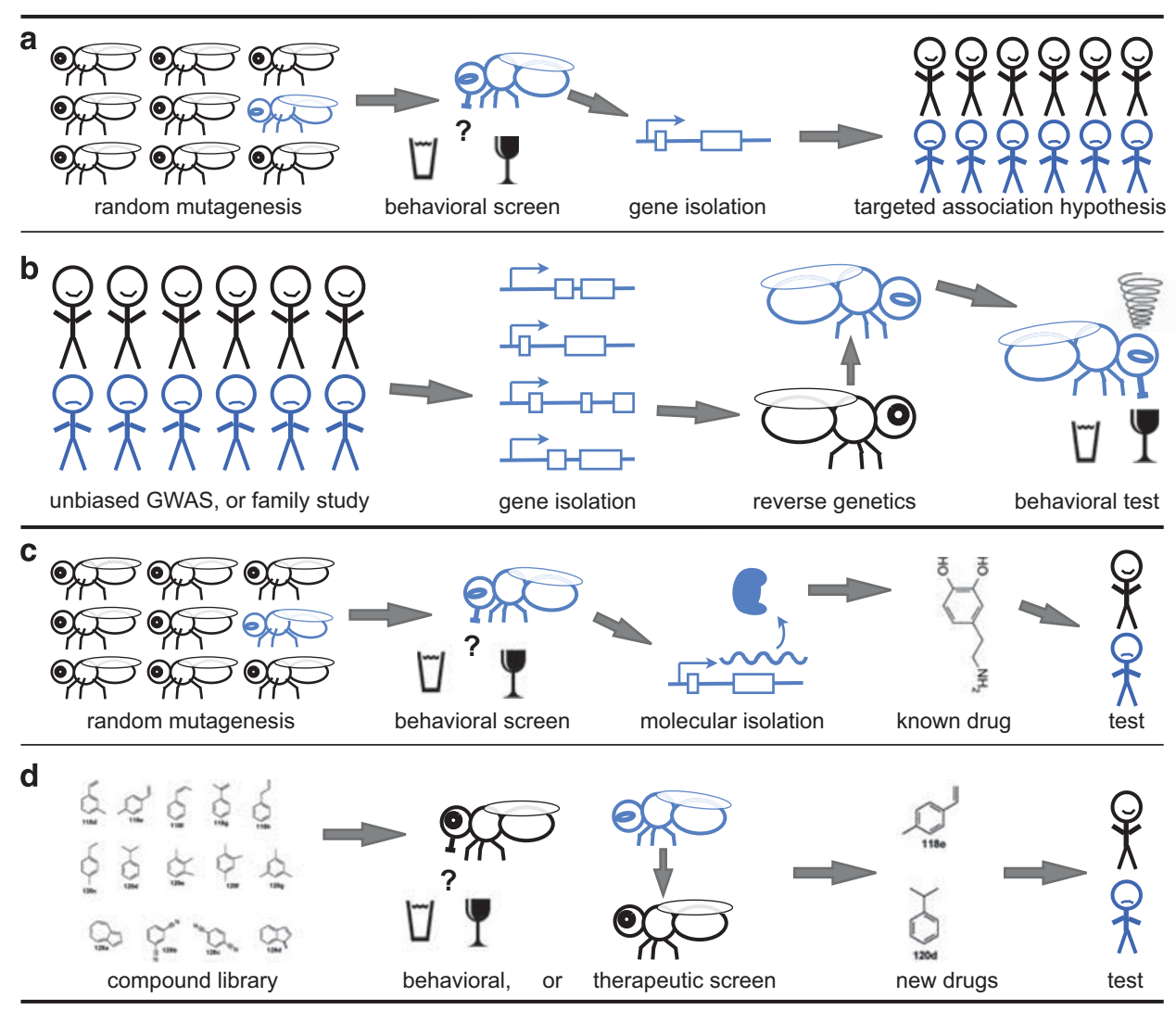

Figure I Translational approaches in neuropsychopharmacology using Drosophila. Modeling neuropsychiatric disorders in Drosophila hinges on the establishment of behavioral assays with a reasonable degree of face validity. Once such an assay is at hand, approaches attaining mechanistic validity include (a) the isolation of mutant strains with behavioral phenotypes. Translational validation can include targeted association studies that have greater statistical power than genome-wide studies. (b) Human disease candidate variants can be introduced into the flies and resulting mutants tested for behavioral abnormalities. Predictive validity can come from (c) unbiased genetic screens that yield genes that produce proteins that are targets of known drugs, or from (d) unbiased compound screens that are either applied to normal flies to affect the behavior under study, or that can rescue disease model flies back toward wild type. Both these approaches $(c, d)$ yield drugs that can be tested in higher animal models and eventually in symptomatic patients. Examples for each of these approaches are cited in the text.

\section{WHY AND HOW WE STUDY DROSOPHILA}

In the face of such challenges, why would one even begin to think about the 'lowly' vinegar fly, Drosophila melanogaster, as a useful model organism to study these disorders? The history of the use of Drosophila in modern biological sciences is a rich one, spanning more than a century (Bellen et al, 2010). The concept that heritable traits are carried on chromosomes was first developed in the fly, as well as many other landmark discoveries in genetics (Sturtevant, 1967). Despite the arthropod lineage being separated from the vertebrate lineage more than 600 million years ago (Adoutte et al, 2000; Peterson et al, 2004), many basic biological, physiological, and neurological properties are conserved between mammals and Drosophila (Edgar and Lehner, 1996; Rubin et al, 2000). This began to be realized in the 1980s, when many developmental genes were discovered in the fly that specify the body plan of this insect. Even though the morphology of the fly differs substantially from mammalian ones, the genes involved in these processes are highly conserved (Gehring et al, 2009), and one can even induce complete ectopic fly eyes by misexpressing a mammalian eye development gene (Halder et al, 1995).
What experimental characteristics and approaches (see Figure 1), then, make vinegar flies a versatile model organism to study? Drosophila is easy and inexpensive to maintain in the laboratory; it can give rise to a large number of genetically identical progeny; and it has a short generation cycle of $<2$ weeks. This has made the fly a prime organism to perform forward genetic screens, where large populations of genetically diverse flies are screened for a particular phenotype of interest, and then the mutant genes responsible are isolated and characterized. The advantages of forward genetic screens are that they are unbiased, and thus shed new insight into the physiological mechanism under study without making assumptions as to its mechanistic underpinnings. We discuss a classic example of successful forward genetics in the following section on circadian rhythms. In addition, forward genetic screens can also be tailored to yield more insight into specific mechanisms. In these modifier screens, one starts with a specific mutant fly strain that has a phenotype, and then screens for additional mutations that (specifically) modify the original phenotype. Proper design of such a gene-by-gene interaction screen will thus allow for the identification of other molecular players in a mechanistically linked pathway under study. Although such screens are also feasible in vertebrates, they are exceedingly rare. Yet, 

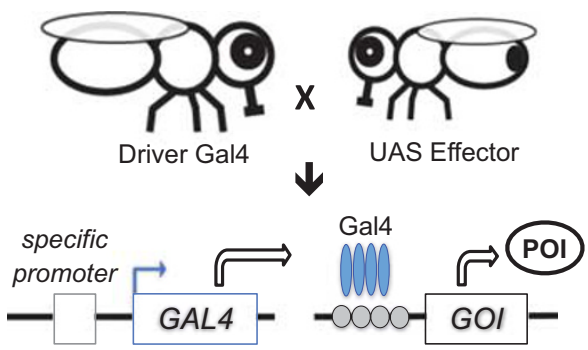

Ubiquitous

Inducible

Tissue (eg. neurons)

Cell-type (eg. dopamine)

cDNA, incl. tags

RNAi for knock-down

Markers (eg. GFP)

Neural effector (eg

Channelrhodopsin

Figure 2 The uses of the Gal4/UAS system. The yeast transcriptional activator Gal4 can be used to regulate gene expression in Drosophila by inserting the gene of interest (GOl) downstream to the UAS (upstream activating sequence). There are now large collections of lines that express GAL4 in a wide variety of cell-type, tissue-specific, temporal, or inducible patterns. Therefore, the expression of the GOI (cDNA, including tags such as HA or fluorescent markers such as GFP; human gene; RNAi for transcript knockdown) can be driven in any of these patterns by crossing the appropriate Gal4 driver line to flies that carry the UAS-GOI transgene to express the protein of interest (POI). In addition, numerous neuronal effectors can be driven by these specific Gal4 drivers, including cell markers and neuronal activators or silencers.

they are often used in Drosophila to expand our understanding of molecular pathways operating to regulate specific processes, even including drug responses (Peru y Colon de Portugal et al, 2012).

After mutant fly strains have been screened and isolated, determining the genes causing the phenotypes has been greatly facilitated by knowing the full sequence of the Drosophila genome that encodes for a little over 16000 genes. It has been estimated that nearly $75 \%$ of diseaserelated genes in humans have functional orthologs in Drosophila (Lloyd and Taylor, 2010; Reiter et al, 2001), reiterating the relevance of the vinegar fly in understanding human disease.

Once a mutant gene is isolated, the gold standard in flies is to verify that it is indeed causing the phenotype by performing a rescue experiment that includes reinserting the normal gene version back into the mutant fly's genome via transgenes. This often includes the binary Gal4/UAS system (Figure 2), where the yeast transcriptional activator Gal4 is expressed under the control of defined enhancers and promoters, and then specifically binds to its upstream activating sequence (UAS) target site to drive expression of a second transgene. For a rescue experiment, this would be a wild-type gene in a mutant background, but the Gal4/UAS system can also be used in reverse genetic approaches (from gene to phenotype). This can include a structure/ function analysis to determine relevant domains and amino acids of a protein under study, and it can also be done to (over)express a human gene and/or its disease variant to study any potential phenotypes this might cause. Thus, when a particular gene that is associated with a human disease is identified, its physiological function and mechanism of action can be elucidated by studying the human gene in flies, or by studying its Drosophila ortholog via reverse genetics.
Recent advances in molecular biology, such as collections of inhibitory RNAs (RNAi) against every fly gene (Dietzl et al, 2007) or CRISPR-mediated knockouts (Gratz et al, 2013), have made reverse genetic approaches in flies much more feasible and expedient, such that even whole gene families can be studied (Chan et al, 2011). Although genetic screens in mice are certainly possible (Kumar et al, 2011), the ease of forward and reverse genetics, coupled with the added advantage of extensive reagent availability at stock centers, make Drosophila approaches both magnitudes faster and also much less expensive. Although such economy of scale can also be achieved in other organisms (like yeast or worms), it is the surprisingly sophisticated behavioral repertoire that makes flies a useful model organism to study neuropsychiatric illnesses.

\section{CONSERVED CLOCKS GUIDE DAILY RHYTHMS}

The brain of Drosophila contains $\sim 200000$ neurons intermediate in neural complexity, in logarithmic terms, between humans and yeast, as the late Seymour Benzer liked to point out (Benzer, 1967). Although he started the field of behavioral genetics by studying simple sensory mechanisms that expressed themselves in behavior (eg, running toward a light), his lab soon expanded to more sophisticated behaviors such as olfactory associative conditioning (Quinn et al, 1974), the social behaviors associated with fly courtship and mating (Hall, 1977), and circadian rhythms (Konopka and Benzer, 1971). The latter serves as a particularly illuminating example of the advances Drosophila can yield, even into understanding complex behaviors. Drosophila has sleep/wake cycles with obvious similarities (and face validity) to human circadian rhythms. The free-running (ie, absent environmental cues) period length of the cycles is $\sim 23.8 \mathrm{~h}$, about a day (circa-dian), but not exactly, underscoring that there is an internal clock driving these rhythms. Similar to people, flies can undergo jet lag (Rothenfluh et al, 2000) when shifted to other times zones, and rhythms out of sync with the environment (akin to shift work) are bad for Drosophila fitness (Klarsfeld and Rouyer, 1998). Konopka and Benzer (1971) were looking for flies that had altered sleep/wake cycles, and in a screen of different Drosophila variants, they came up with three different alleles of the same gene, period, that came in a short-period $19 \mathrm{~h}$ flavor, a longperiod $29 \mathrm{~h}$ variant, and an arrhythmic allele that caused flies to have sleep/wake cycles with no rhyme or reason. It took 15 years to molecularly isolate the period gene, but eventually it was determined that the period gene is central to an autoregulatory feedback loop where period gets transcribed; RNA, and later, protein accumulate; the protein then translocates into the nucleus to repress its own transcription; and when the protein is finally degraded, the cycle starts anew (Hardin et al, 1990). For a long time it remained unclear whether period was unique to Drosophila, and the cloning of any mammalian period gene proved challenging. In the 1990s, there was an explosion of insight and publications describing mammalian orthologs of period as well as numerous additional, conserved molecular components of the core clock mechanism, all centered around a similar negative feedback loop (Allada et al, 2001; Young and Kay, 2001). In addition, mutations in patients suffering from 
sleep disturbances, such as familial advanced sleep phase syndrome, have been mapped to those very genes that were initially isolated and characterized in Drosophila (Toh et al, 2001). Overall, it emerged that there is very strong mechanistic validity in studying circadian rhythms in Drosophila, and many of the conserved components of the clock were first isolated, and their mechanisms elucidated, in flies.

Disturbances in sleep/wake rhythms are a common comorbidity in many psychiatric patients, and circadian abnormalities, including mutations in core clock genes, are associated with mood disorders, including depression and bipolar disorder (McClung, 2007). In addition, animals with defective core clock function show alterations in their responses to drugs of abuse (Spanagel et al, 2005), including Drosophila (Andretic et al, 1999; Pohl et al, 2013). Clock genes thus regulate processes beyond circadian rhythmicity, including behavioral responses to drugs of abuse.

\section{STUDYING DRUGS OF ABUSE WITH FLIES}

Addiction is probably the neuropsychiatric disorder that has been modeled with most face validity in flies. Drug addiction is mostly characterized by compulsive drug seeking and use, despite the harmful consequences. There are two broad categories: substance abuse and substance dependence. Substance abuse is defined as continued drug use despite physically hazardous situations or legal or interpersonal problems, whereas substance dependence includes physical symptoms like withdrawal and tolerance as well as uncontrolled use. One of the two most commonly used rodent models to ask whether rats and mice like specific drugs is conditioned place preference (CPP), where a drug is applied as a reinforcer in a specific context, and animals will then prefer to spend time in the context/place in which drugs were applied over the other, neutral control context/place. The other assay is self-administration that may be a leverpress-mediated injection of drug or choosing to drink alcohol in a two-bottle choice paradigm. These models have obvious face value, as the first shows that the animals 'like' the drugs and they form positive associations upon encountering it, and the second also adds voluntary drug intake as a characteristic.

Alcohol is one of the most widely used and abused psychoactive drugs in the world. Acute ethanol exposure can result in both short-term behavioral impairment and longterm addiction. In the lab, flies are often exposed to ethanol in streams of defined ethanol vapor to measure their behavioral responses, surprisingly similar to mammalian responses. At low doses, flies show locomotor activation (Wolf et al, 2002) and disinhibition (Lee et al, 2008), whereas higher doses lead to loss of righting reflex and sedation (Rodan et al, 2002). These behaviors can be measured quantitatively by visual observation and video tracking (Rothenfluh et al, 2006; Wolf et al, 2002), and numerous genes have been isolated that affect these processes (reviewed in Kaun et al, 2012; Rodan and Rothenfluh, 2010). Although resistance to the intoxicating and sedating effects of ethanol is a risk factor for the development of alcoholism (Schuckit et al, 2004), it is not a diagnostic criterion for alcohol abuse, and therefore studying sedation in flies lacks some face validity for modeling abuse. For this reason, additional paradigms have been developed over the past years. First, it was shown that flies, similar to mammals, develop tolerance to repeat exposures of ethanol. Thus, with repeated exposures the dose of ethanol needs to be increased to attain the same behavioral end point because flies acquire resistance to the effect of ethanol intoxication. This tolerance is an adaptation in the nervous system, and thus called functional tolerance, and not a change in the kinetics of ethanol absorption and metabolism (Scholz et al, 2000). Recovery from ethanol pre-exposure in flies can also lead to withdrawal symptoms, such as seizure susceptibility (Ghezzi et al, 2012). In addition, ethanol vapor can act as a reinforcer in an assay similar to rodent CPP, where a neutral odor gains attractiveness after having been paired with a locomotor activating dose of ethanol exposure (Kaun et al, 2011). Finally, in a two-bottle choice paradigm, flies develop preference for a sucrose/yeast solution containing 15\% ethanol over the span of a few days, and they will even overcome a bitter tasting compound in the solution in order to drink (Devineni and Heberlein, 2009). A similar consumption preference assay has recently been scaled for higher throughput and allows for screening of mutant flies with changes in experience-dependent ethanol consumption preference (Peru y Colon de Portugal et al, 2014).

These novel assays provide more obvious face validity to the study of drugs of abuse in flies, but what have we learned from flies that speaks to the mechanistic validity of the system? We will briefly highlight three lines of experiments that clearly illuminate the translational value of studying alcohol-induced behavior in flies. The first study identified the autism susceptibility candidate 2 gene (AUTS2) in a human genome-wide association study for alcohol consumption (Schumann et al, 2011). Experiments to validate this finding in humans are obviously constrained, and because of the ready availability of these mutations in flies, combined with fast breeding time, the authors tested three different mutations in the fly ortholog tay for their ethanolinduced behaviors. They found that all three caused resistance to ethanol-induced sedation (Schumann et al, 2011), suggesting that AUTS2 is involved in regulating ethanol-induced behaviors across species, and highlighting the utility of Drosophila as a reverse genetic model to behaviorally validate candidate genes found in people.

Unlike other drugs, ethanol acts on multiple targets and can affect many different ion channels and other molecular targets (Koob, 2004). Numerous proteins and pathways targeted may not be known yet, thus making unbiased forward genetic approaches particularly useful to implicate novel genes in ethanol-induced responses. One novel gene recently implicated in ethanol consumption is RSU1, and its fly ortholog icarus (ics). Mutants in ics were isolated in a forward genetic screen because of their resistance to ethanolinduced sedation. A functional ics gene is required in the adult nervous system of the fly for normal ethanol-induced behavior. Furthermore, ethanol-naive flies lacking all ics function show immediate ethanol preference in a two-bottle choice paradigm (where normal flies take 2-3 days to acquire preference; Ojelade et al, 2015). Because fly acquisition of preference likely involves reward-related processes, the 70 SNPs in human RSU1 were tested for association with altered brain activity during reward anticipation using 
functional imaging. One SNP showed significant association with increased activation in the ventral striatum. Because that SNP is localized in an intron, it was hypothesized to be a marker for an unidentified linked causal variant in the vicinity. An analysis of the group of 22 SNPs in linkage disequilibrium recapitulated its association with ventral striatum activation, and also found significant associations with the frequency of lifetime drinking in the same cohort, and with alcohol dependence in a second, independent cohort (Ojelade et al, 2015). Rsu1/ics thus regulates ethanol consumption in Drosophila and humans. Molecular insight into how this might work was garnered from flies, where Rsul acts in a signaling cascade downstream of the integrin cell adhesion molecule, and upstream of the small GTPase Rac1 to regulate the actin cytoskeleton (Ojelade et al, 2015). Both integrin (Kramár et al, 2006) and Rac1 (Rothenfluh and Cowan, 2013) are involved in synaptic plasticity mechanisms that likely underlie the behavioral phenotypes observed. Three general lessons can be drawn from this study. First, forward genetics in flies can identify novel genes that also have relevance to human behavior. Second, isolation of such genes in flies allows for targeted hypothesis testing with human subjects and samples. By eliminating most of the multiple comparisons demanded by genome-wide studies, this leads to much greater statistical power, and a higher likelihood of finding statistically significant association. Finally, elucidating the molecular mechanism in which a given gene, such as RSU1/ics, participates can be readily achieved in flies, including establishing in vivo behavioral relevance. This provides mechanistic insight into ethanol consumption preference in flies, and yet more testable hypotheses for translation to human studies.

In a third set of experiments, mutations in the Sterile-20 family kinase gene Happy Hour (Hppy) were isolated because of their resistance to ethanol-induced sedation (Corl et al, 2009). Hppy acts in the fly nervous system, and a number of genetic interactions experiments placed it in the epidermal growth factor receptor (EGFR) pathway, with increased EGFR signaling causing resistance to ethanol-induced sedation. This pathway is well studied in the context of cell growth and cancer, and erlotinib and gefitinib are two clinically used EGFR inhibitors. When adult flies were fed either of those two drugs, they became more sensitive to ethanol-induced sedation, just as hypothesized from the genetic data. The authors then fed erlotinib to ethanolconsuming rats, and found that it reduced ethanol, but not sucrose or water intake (Corl et al, 2009). Gene identification from an unbiased genetic screen was therefore translated to medication-mediated reduction of ethanol drinking. Overall, these three studies clearly highlight the translational potential of Drosophila, and they show that achieving face, mechanistic, and even predictive validity of this model organism can be attained in the study of certain neuropsychiatric disorders.

\section{FRAGILE X PROTEIN AND ASD}

ASD is a neurodevelopmental condition characterized by impaired social interaction and communication as well as repetitive behavior. Autism has a strong genetic basis, although a complex one (Sanders et al, 2012). ASD and several rare monogenetic neurodevelopmental disorders are sometimes grouped together, as they have complex neurological symptoms, including cognitive dysfunction. One of the best-studied autism genes is FMR1 that is also responsible for causing fragile $\mathrm{X}$ syndrome (FXS). The degree of cognitive deficits observed in fragile $\mathrm{X}$ patients ranges from mild learning disabilities to severe mental retardation, with progressive cognitive decline occurring with time. The presentations of ASD that have been modeled with some face validity are decreased cognitive function and repetitive behaviors. Mutations in $d F m r 1$, which shows high homology to the mammalian gene, lead to repetitive grooming in flies (Tauber et al, 2011) and to reduced memory in a social conditioning paradigm (McBride et al, 2005). Indeed, these mutant flies have even been shown to display reduced courtship, the social behavior that leads to mating. In flies, this consists of a ritual of successive behavioral steps (and advances), ultimately (after minutes of courting) leading to copulation. Males lacking $d F m r 1$ do show some courtship, but they often fail to progress to the next step in the ritual, and 'lose interest' (Dockendorff et al, 2002). One could thus argue that even in the domain of reduced social interaction, the Drosophila model possesses some face validity.

Fly $d F m r 1$ mutants also show many other similarities to mouse mutants in FMR1, including structural changes in individual neurons such as axonal and dendritic branches (Callan and Zarnescu, 2011; McBride et al, 2012; van Alphen and van Swinderen, 2013; Zhang and Broadie, 2005). This mechanistic validity is further extended by findings that FMR1 knockout mice show defects in metabotropic glutamate receptor (mGluR) signaling that has been linked to several phenotypes that are similar to fragile $\mathrm{X}$ symptoms, resulting in the hypothesis that a significant portion of the disease phenotypes are due to mGluR dysregulation (Bear et al, 2004). Consistent with this hypothesis, McBride et al (2005 treated dFmr1 mutant flies with different mGluR antagonists and could rescue the behavioral and the structural deficits found in these $d F m r 1$ mutant flies back towards wild type. Their study extends the model into predictive validity and the search for novel therapeutic strategies was pushed even further in a screen to rescue dFmr1 mutants with a library of 2000 different chemical compounds (Chang et al, 2008). The isolated compounds, mostly targeting GABA-ergic signaling, have thus revealed novel pathways regulating $\mathrm{dFmr} 1$ function in vivo, expanding the avenues of traditional drug discovery approaches.

\section{OTHER DISORDERS}

Certain disorders are never going to have any face validity in flies, such as suicidal impulses. This caveat, however, applies to all animal models of some human disorders. One might be hard pressed to model the positive symptoms of schizophrenia (SZ), such as hallucinations, in animals. Because this might be similarly hard for the negative symptoms, such as lack of affect, one approach to model this disorder is to focus on mechanistic validity, and to molecularly study a SZ-associated human gene in flies. Expression of human DISC1, one such SZ-associated gene, in the mushroom bodies of flies leads to an increased sleep phenotype in the transgenic flies. Disrupted sleep/wake cycles are found in 
many SZ patients, and Sawamura et al (2008) used human DISC1 transgenic flies to tease out molecular mechanisms resulting in the sleep disorder. They found that transgenic flies with accumulation of exogenous human DISC1 in the nucleus exhibited disturbances in sleep homeostasis that they associated with CREB signaling/CRE-mediated gene transcription. Therefore, although the sleep phenotype alone may seem to inadequately model a complex disorder such as SZ, this phenotype may be sufficient for high-throughput reverse genetic screens that can test thousands of candidates genes associated with schizophrenia to gain mechanistic insight into how these genes function.

Because SZ is highly heritable (Tandon et al, 2008), one might be tempted to use a genetically tractable organism such as flies to model phenotypes associated with it. As mentioned above, overt symptoms such as hallucinations or psychosis are difficult to model in animals. Endophenotypes, which are intermediate between the symptomatic presentation and underlying (genetic) etiology (Insel and Cuthbert, 2009), are another approach to unravel the mechanisms leading to psychiatric disorders. For SZ, working memory or sensorimotor gating deficits such as prepulse inhibition and habituation are such endophenotypes. As they are more clearly defined and quantifiable, they are more easily amenable to modeling in animals. Flies can habituate to many different innocuous stimuli (Engel and $\mathrm{Wu}, 2009$ ), but the more commonly studied prepulse inhibition has not been shown to exist in flies (yet). We say this because another invertebrate, the sea slug Tritonia, does show reproducible, quantifiable prepulse inhibition of its escape response (Mongeluzi et al, 1998). Describing this phenomenon in Drosophila thus likely awaits the right investigators, with the right inspiration to establish a quantifiable behavior. Elucidating the mechanisms governing such endophenotypes may thus yield insight into the genetic causes, or at least risk factors, of complex diseases such as SZ. Similarly, as the NIMH Research Domain Criteria (RDoC) Project is defining quantifiable biological measures for clinical research and disease phenotyping (Simmons and Quinn, 2014), these specified measures and tests might be more easily modeled in animals, and aid in establishing the mechanistic etiologies of various traits underlying numerous psychiatric disorders.

These questions and considerations similarly hold true for modeling other disorders such as depression. Can flies be depressed? They fight, and highly aggressive strains have been isolated (Penn et al, 2010). One could imagine that normal flies could be chronically defeated by these 'bullies', similar to the chronic social defeat depression model in mice (Krishnan et al, 2007). If they are 'sexually frustrated' and are prevented from mating, they drink more alcohol (Shohat-Ophir et al, 2012). Recently, Yang et al (2013) have shown that flies develop learned helplessness, an established rodent model for depression. Because of the ease of genetic manipulation, including the reverse genetic approaches of testing known genes linked to specific human neuropsychiatric disorders, finding the right behavioral assay with a semblance of face validity thus often remains the initial challenge in modeling such disorders in flies. Once found though, molecular advances and insights can rapidly follow. This expands the usefulness of flies into learning about mechanisms and suggesting targets for therapeutics, or even therapeutics themselves, as done with a drug screen to find compounds altering monoaminergic deficiencies in flies (Lawal et al, 2014). Many neuropsychiatric disorders such as $\mathrm{ADHD}$, depression, and addiction affect monoaminergic pathways, thereby making them a promising target for the development of novel screening approaches. This includes targeting presynaptic proteins required for the release of monoamines such as the vesicular monoamine transporter (VMAT), required for transport of all amines into synaptic vesicles. Modifier screens in Drosophila provide a new strategy to identify novel neuropsychiatric drugs, and Lawal et al (2014) performed a successful drug screen to find compounds altering monoaminergic deficiencies in flies. This example further underscores the utility of flies for testing genetic hypotheses as well as for generating genetic and even therapeutic hypotheses.

\section{OUTLOOK}

The field of Drosophila neurogenetics has changed dramatically over the past 20 years. The focus of most of this research community used to be centered around the molecular mechanisms of development such as axon pathfinding or neuronal cell-fate decisions, and relatively few scientists concentrated on behavior. However, as behavioral assays have become much more sophisticated and quantifiable, together with the ability to obtain more subtle, or adult-specific mutations, a large number of research labs now incorporate behavioral readouts into their arsenal. This has led to unbiased approaches trying to understand fundamental behaviors and their plasticity, yielding gene variants with a human connection. In addition, more labs have ventured into specifically modeling human disorders, and studying genes with known links to neuropsychiatric disorders. Certain disorders may never be modeled in flies, such as the ones affecting those parts of human brain anatomy/function that are clearly distinct. This may include prefrontal cortical diseases (do flies have a cortex?) or addiction to nicotine (whose receptors in flies mediate major excitatory neurotransmission in the brain; hence flies exposed to nicotine have seizures). However, fly researchers are developing an increasingly precise anatomical toolkit that allows for Gal4-mediated gene manipulation in ever more spatially restricted neurons. In addition, many new tools, like optogenetics, allow for the precise functional manipulation of these circuits. This will enable us to test how much functional homology is there between the Drosophila and human brain (in addition to the proposed deep evolutionary developmental homology; Strausfeld and Hirth, 2013). Although we will never have the ability to ask the fly 'how did that make you feel?,' if the past is any indication, the future will allow us to model human conditions with ever more increasing face, mechanistic, and even predictive validity. In addition, as flies will continue to believe that they can fly, we might increasingly understand not just the how but even the why.

\section{FUNDING AND DISCLOSURE}

ASN has been working for the Boston Consulting Group in Dallas since the beginning of 2015. The authors declare no conflict of interest. 


\section{ACKNOWLEDGMENTS}

This work was funded by the NIH, R01AA019526 and R21AA022404. We thank Aylin Rodan for critical reading of the manuscript.

\section{REFERENCES}

Adoutte A, Balavoine G, Lartillot N, Lespinet O, Prud'homme B, de Rosa R (2000). The new animal phylogeny: reliability and implications. Proc Natl Acad Sci USA 97: 4453-4456.

Alaerts M, Del-Favero J (2009). Searching genetic risk factors for schizophrenia and bipolar disorder: learn from the past and back to the future. Hum Mutat 30: 1139-1152.

Allada R, Emery P, Takahashi JS, Rosbash M (2001). Stopping time: the genetics of fly and mouse circadian clocks. Annu Rev Neurosci 24: 1091-1119.

Andretic R, Chaney S, Hirsh J (1999). Requirement of circadian genes for cocaine sensitization in Drosophila. Science 285: 1066-1068.

Bear MF, Huber KM, Warren ST (2004). The mGluR theory of fragile X mental retardation. Trends Neurosci 27: 370-377.

Bellen HJ, Tong C, Tsuda H (2010). 100 years of Drosophila research and its impact on vertebrate neuroscience: a history lesson for the future. Nat Rev Neurosci 11: 514-522.

Benzer S (1967). Behavioral mutants of Drosophila isolated by countercurrent distribution. Proc Natl Acad Sci USA 58: $1112-1119$.

Callan MA, Zarnescu DC (2011). Heads-up: new roles for the fragile $\mathrm{X}$ mental retardation protein in neural stem and progenitor cells. Genesis 49: 424-440.

Chan CC, Scoggin S, Wang D, Cherry S, Dembo T, Greenberg B et al (2011). Systematic discovery of Rab GTPases with synaptic functions in Drosophila. Curr Biol 21: 1704-1715.

Chang S, Bray SM, Li Z, Zarnescu DC, He C, Jin P et al (2008). Identification of small molecules rescuing fragile $\mathrm{X}$ syndrome phenotypes in Drosophila. Nat Chem Biol 4: 256-263.

Corl AB, Berger KH, Ophir-Shohat G, Gesch J, Simms JA, Bartlett SE et al (2009). Happyhour, a Ste20 family kinase, implicates EGFR signaling in ethanol-induced behaviors. Cell 137: 949-960.

Devineni AV, Heberlein U (2009). Preferential ethanol consumption in Drosophila models features of addiction. Curr Biol 19: $2126-2132$.

Dietzl G, Chen D, Schnorrer F, Su KC, Barinova Y, Fellner M et al (2007). A genome-wide transgenic RNAi library for conditional gene inactivation in Drosophila. Nature 448: 151-156.

Dockendorff TC, Su HS, McBride SM, Yang Z, Choi CH, Siwicki KK et al (2002). Drosophila lacking dfmr1 activity show defects in circadian output and fail to maintain courtship interest. Neuron 34: 973-984.

Edgar BA, Lehner CF (1996). Developmental control of cell cycle regulators: a fly's perspective. Science 274: 1646-1652.

Engel JE, Wu C-F (2009). Neurogenetic approaches to habituation and dishabituation in Drosophila. Neurobiol Learn Mem 92: 166-175.

Gehring WJ, Kloter U, Suga H (2009). Evolution of the Hox gene complex from an evolutionary ground state. Curr Top Dev Biol 88: 35-61.

Ghezzi A, Krishnan HR, Atkinson NS (2012). Susceptibility to ethanol withdrawal seizures is produced by $\mathrm{BK}$ channel gene expression. Addict Biol 19: 332-337.

Gratz SJ, Wildonger J, Harrison MM, O'Connor-Giles KM (2013). CRISPR/Cas9-mediated genome engineering and the promise of designer flies on demand. Fly 7: 249-255.

Halder G, Callaerts P, Gehring WJ (1995). Induction of ectopic eyes by targeted expression of the eyeless gene in Drosophila. Science 267: 1788-1792.
Hall JC (1977). Portions of the central nervous system controlling reproductive behavior in Drosophila melanogaster. Behav Genet 7: 291-312.

Hardin PE, Hall JC, Rosbash M (1990). Feedback of the Drosophila period gene product on circadian cycling of its messenger RNA levels. Nature 343: 536-540.

Insel TR, Cuthbert BN (2009). Endophenotypes: bridging genomic complexity and disorder heterogeneity. Biol Psychiatry 66: 988-989.

Iyengar SK, Elston RC (2007). The genetic basis of complex traits: rare variants or "common gene, common disease"? Methods Mol Biol 376: 71-84.

Kaun KR, Azanchi R, Maung Z, Hirsh J, Heberlein U (2011). A Drosophila model for alcohol reward. Nat Neurosci 14: 612-619.

Kaun KR, Devineni AV, Heberlein U (2012). Drosophila melanogaster as a model to study drug addiction. Human Genet 131: 959-975.

Klarsfeld A, Rouyer F (1998). Effects of circadian mutations and LD periodicity on the life span of Drosophila melanogaster. J Biol Rhythms 13: 471-478.

Konopka RJ, Benzer S (1971). Clock mutants of Drosophila melanogaster. Proc Natl Acad Sci USA 68: 2112-2116.

Koob GF (2004). A role for GABA mechanisms in the motivational effects of alcohol. Biochem Pharmacol 68: 1515-1525.

Kramár EA, Lin B, Rex CS, Gall CM, Lynch G (2006). Integrindriven actin polymerization consolidates long-term potentiation. Proc Natl Acad Sci USA 103: 5579-5584.

Krishnan V, Han M-H, Graham DL, Berton O, Renthal W, Russo SJ et al (2007). Molecular adaptations underlying susceptibility and resistance to social defeat in brain reward regions. Cell 131: 391-404.

Kumar V, Kim K, Joseph C, Thomas LC, Hong H, Takahashi JS (2011). Second-generation high-throughput forward genetic screen in mice to isolate subtle behavioral mutants. Proc Natl Acad Sci USA 108: 15557-15564.

Lawal HO, Terrell A, Lam HA, Djapri C, Jang J, Hadi R et al (2014). Drosophila modifier screens to identify novel neuropsychiatric drugs including aminergic agents for the possible treatment of Parkinson's disease and depression. Mol Psychiatry 19: 235-242.

Lee HG, Kim YC, Dunning JS, Han KA (2008). Recurring ethanol exposure induces disinhibited courtship in Drosophila. PLoS One 3: e1391.

Lloyd TE, Taylor JP (2010). Flightless flies: Drosophila models of neuromuscular disease. Ann NY Acad Sci 1184: e1-e20.

McBride SM, Bell AJ, Jongens TA (2012). Behavior in a Drosophila model of fragile X. Results Prob Cell Differ 54: 83-117.

McBride SM, Choi CH, Wang Y, Liebelt D, Braunstein E, Ferreiro D et al (2005). Pharmacological rescue of synaptic plasticity, courtship behavior, and mushroom body defects in a Drosophila model of fragile X syndrome. Neuron 45: 753-764.

McClung CA (2007). Circadian genes, rhythms and the biology of mood disorders. Pharmacol Ther 114: 222-232.

Mongeluzi DL, Hoppe TA, Frost WN (1998). Prepulse inhibition of the Tritonia escape swim. J Neurosci 18: 8467-8472.

Ojelade SA, Jia T, Rodan AR, Chenyang T, Kadrmas JL, Cattrell A et al (2015). Rsul regulates ethanol consumption in Drosophila and humans. Proc Natl Acad Sci USA 112: E4085-E4093.

Penn JK, Zito MF, Kravitz EA (2010). A single social defeat reduces aggression in a highly aggressive strain of Drosophila. Proc Natl Acad Sci USA 107: 12682-12686.

Peru y Colon de Portugal RL, Acevedo SF, Rodan AR, Chang LY, Eaton BA, Rothenfluh A (2012). Adult neuronal Arf6 controls ethanol-induced behavior with Arfaptin downstream of Rac1 and RhoGAP18B. J Neurosci 32: 17706-17713.

Peru y Colon de Portugal RL, Ojelade SA, Penninti PS, Dove RJ, Nye MJ, Acevedo SF et al (2014). Long-lasting, experiencedependent alcohol preference in Drosophila. Addict Biol 19: 392-401. 
Peterson KJ, Lyons JB, Nowak KS, Takacs CM, Wargo MJ, McPeek MA (2004). Estimating metazoan divergence times with a molecular clock. Proc Natl Acad Sci USA 101: 6536-6541.

Pohl JB, Ghezzi A, Lew LK, Robles RB, Cormack L, Atkinson NS (2013). Circadian genes differentially affect tolerance to ethanol in Drosophila. Alcohol Clin Exp Res 37: 1862-1871.

Quinn WG, Harris WA, Benzer S (1974). Conditioned behavior in Drosophila melanogaster. Proc Natl Acad Sci USA 71: 708-712.

Reiter LT, Potocki L, Chien S, Gribskov M, Bier E (2001). A systematic analysis of human disease-associated gene sequences in Drosophila melanogaster. Genome Res 11: 1114-1125.

Rodan AR, Kiger JA Jr, Heberlein U (2002). Functional dissection of neuroanatomical loci regulating ethanol sensitivity in Drosophila. J Neurosci 22: 9490-9501.

Rodan AR, Rothenfluh A (2010). The genetics of behavioral alcohol responses in Drosophila. Int Rev Neurobiol 91: 25-51.

Rothenfluh A, Cowan CW (2013). Emerging roles of actin cytoskeleton regulating enzymes in drug addiction: actin or reactin'? Curr Opin Neurobiol 23: 507-512.

Rothenfluh A, Threlkeld RJ, Bainton RJ, Tsai LT, Lasek AW, Heberlein U (2006). Distinct behavioral responses to ethanol are regulated by alternate RhoGAP18B isoforms. Cell 127: 199-211.

Rothenfluh A, Young MW, Saez L (2000). A TIMELESSindependent function for PERIOD proteins in the Drosophila clock. Neuron 26: 505-514.

Rubin GM, Yandell MD, Wortman JR, Gabor Miklos GL, Nelson CR, Hariharan IK et al (2000). Comparative genomics of the eukaryotes. Science 287: 2204-2215.

Sanders SJ, Murtha MT, Gupta AR, Murdoch JD, Raubeson MJ, Willsey AJ et al (2012). De novo mutations revealed by whole-exome sequencing are strongly associated with autism. Nature 485: 237-241.

Sawamura N, Ando T, Maruyama Y, Fujimuro M, Mochizuki H, Honjo K et al (2008). Nuclear DISC1 regulates CRE-mediated gene transcription and sleep homeostasis in the fruit fly. Mol Psychiatry 13: 1138-1148, 1069.

Scholz H, Ramond J, Singh CM, Heberlein U (2000). Functional ethanol tolerance in Drosophila. Neuron 28: 261-271.

Schuckit MA, Smith TL, Kalmijn J (2004). Findings across subgroups regarding the level of response to alcohol as a risk factor for alcohol use disorders: a college population of women and Latinos. Alcohol Clin Exp Res 28: 1499-1508.
Schumann G, Coin LJ, Lourdusamy A, Charoen P, Berger KH, Stacey D et al (2011). Genome-wide association and genetic functional studies identify autism susceptibility candidate 2 gene (AUTS2) in the regulation of alcohol consumption. Proc Natl Acad Sci USA 108: 7119-7124.

Shohat-Ophir G, Kaun KR, Azanchi R, Mohammed H, Heberlein U (2012). Sexual deprivation increases ethanol intake in Drosophila. Science 335: 1351-1355.

Simmons JM, Quinn KJ (2014). The NIMH Research Domain Criteria (RDoC) Project: implications for genetics research. Mamm Genome 25: 23-31.

Spanagel R, Pendyala G, Abarca C, Zghoul T, Sanchis-Segura C, Magnone MC et al (2005). The clock gene Per2 influences the glutamatergic system and modulates alcohol consumption. Nat Med 11: 35-42.

Strausfeld NJ, Hirth F (2013). Deep homology of arthropod central complex and vertebrate basal ganglia. Science 340: 157-161.

Sturtevant A (1967). A History of Genetics. Cold Spring Harbor Laboratory Press: Cold Spring Harbor, NY.

Tandon R, Keshavan MS, Nasrallah HA (2008). Schizophrenia, "just the facts" what we know in 2008. 2. Epidemiology and etiology. Schizophr Res 102: 1-18.

Tauber JM, Vanlandingham PA, Zhang B (2011). Elevated levels of the vesicular monoamine transporter and a novel repetitive behavior in the Drosophila model of fragile X syndrome. PLoS One 6: e27100.

Toh KL, Jones CR, He Y, Eide EJ, Hinz WA, Virshup DM et al (2001). An hPer2 phosphorylation site mutation in familial advanced sleep phase syndrome. Science 291: 1040-1043.

van Alphen B, van Swinderen B (2013). Drosophila strategies to study psychiatric disorders. Brain Res Bull 92: 1-11.

Wolf FW, Rodan AR, Tsai LT, Heberlein U (2002). High-resolution analysis of ethanol-induced locomotor stimulation in Drosophila. J Neurosci 22: 11035-11044.

Yang Z, Bertolucci F, Wolf R, Heisenberg M (2013). Flies cope with uncontrollable stress by learned helplessness. Curr Biol 23: 799-803.

Young MW, Kay SA (2001). Time zones: a comparative genetics of circadian clocks. Nat Rev Genet 2: 702-715.

Zhang YQ, Broadie K (2005). Fathoming fragile X in fruit flies. Trends Genet 21: 37-45. 